\title{
Genes CEP55, FOXD3, FOXF2, GNAO1, GRIA4, and KCNA5 as potential diagnostic biomarkers in colorectal cancer
}

\author{
Nina Hauptman ${ }^{1 *}$ DD, Daša Jevšinek Skok ${ }^{1,2}$, Elena Spasovska ${ }^{1}$, Emanuela Boštjančič ${ }^{2}$ and Damjan Glavač
}

\begin{abstract}
Background: Colorectal cancer (CRC) is one of the leading causes of death by cancer worldwide and in need of novel potential diagnostic biomarkers for early discovery.

Methods: We conducted a two-step study. We first employed bioinformatics on data from The Cancer Genome Atlas to obtain potential biomarkers and then experimentally validated some of them on our clinical samples. Our aim was to find a methylation alteration common to all clusters, with the potential of becoming a diagnostic biomarker in CRC.

Results: Unsupervised clustering of methylation data resulted in four clusters, none of which had a known common genetic or epigenetic event, such as mutations or methylation. The intersect among clusters and regulatory regions resulted in 590 aberrantly methylated probes, belonging to 198 differentially expressed genes. After performing pathway and functional analysis on differentially expressed genes, we selected six genes: CEP55, FOXD3, FOXF2, GNAO1, GRIA4 and KCNA5, for further experimental validation on our own clinical samples. In silico analysis demonstrated that CEP55 was hypomethylated in $98.7 \%$ and up-regulated in $95.0 \%$ of samples. Genes FOXD3, FOXF2, GNAO1, GRIA4 and KCNA5 were hypermethylated in 97.9, 81.1, 80.3, 98.4 and 94.0\%, and downregulated in $98.3,98.9,98.1,98.1$ and $98.6 \%$ of samples, respectively. Our experimental data show CEP55 was hypomethylated in $97.3 \%$ of samples and down-regulated in all samples, while FOXD3, FOXF2, GNAO1, GRIA4 and KCNA5 were hypermethylated in 100.0, 90.2, 100.0, 99.1 and 100.0\%, and down-regulated in 68.0, 76.0, 96.0, 95.2 and $84.0 \%$ of samples, respectively. Results of in silico and our experimental analyses showed that more than $97 \%$ of samples had at least four methylation markers altered.

Conclusions: Using bioinformatics followed by experimental validation, we identified a set of six genes that were differentially expressed in CRC compared to normal mucosa and whose expression seems to be methylation dependent. Moreover, all of these six genes were common in all methylation clusters and mutation statuses of CRC and as such are believed to be an early event in human CRC carcinogenesis and to represent potential CRC biomarkers.
\end{abstract}

Keywords: Colorectal cancer, Methylation, Expression, Bioinformatics approach, Experimental validation

\footnotetext{
*Correspondence: nina.hauptman@mf.uni-lj.si

'Institute of Pathology, Faculty of Medicine, University of Ljubljana, Korytkova

2, SI-1000 Ljubljana, Slovenia

Full list of author information is available at the end of the article
}

(c) The Author(s). 2019 Open Access This article is distributed under the terms of the Creative Commons Attribution 4.0 International License (http://creativecommons.org/licenses/by/4.0/), which permits unrestricted use, distribution, and reproduction in any medium, provided you give appropriate credit to the original author(s) and the source, provide a link to the Creative Commons license, and indicate if changes were made. The Creative Commons Public Domain Dedication waiver (http://creativecommons.org/publicdomain/zero/1.0/) applies to the data made available in this article, unless otherwise stated. 


\section{Background}

Colorectal cancer (CRC) is one of the leading causes of death by cancer in both genders [1]. CRC occurs through a process of malignant transformation, when numerous genetic and epigenetic events transform normal colon mucosa to adenocarcinoma [2]. It is a very heterogeneous disease, in which three major molecular pathways have been identified. The most common pathway is the chromosomal instability (CIN) pathway, which is characterized by an accumulation of mutations in specific genes (e.g., APC, KRAS, BRAF, TP53) [2], and accounts for $65-70 \%$ of sporadic CRC cases [3]. The microsatellite instability (MSI) pathway accounts for approximately $15 \%$ of sporadic CRC, and is characterized by deficiency in DNA mismatch repair (MMR) genes (e.g. $M L H 1, M S H 2, M S H 6, P M S 2$ ) [4]. Silencing of MMR genes in the MSI type of CRC occurs through promoter hypermethylation, a common molecular alteration at epigenetic level. In more than $80 \%$ of MSI cases, promoter hypermethylation occurred in the $M L H 1$ gene [5]. The third molecular pathway is the CpG island methylator phenotype (CIMP); an epigenetic instability pathway. One of these three pathways is usually predominant but they are not mutually exclusive $[6,7]$.

CIMP has been extensively studied, not only in CRC but also in bladder, gastric, lung and breast cancer [8]. Some researchers have proposed three CIMP subtypes: CIMP high (CIMP-H), CIMP low (CIMP-L), and non-CIMP subtypes [5]. The CIMP-H subtype is significantly associated with the proximal colon and mutations in gene $B R A F$, whereas the CIMP-L subtype has intermediate methylation levels and is associated with mutations in KRAS gene [9]. Moreover, The Cancer Genome Atlas (TCGA) consortium describes four epigenetic subtypes (CIMP-H, CIMP-L, Cluster 3, and Cluster 4), of which Clusters 3 and 4 are defined as non-CIMP subtypes [10].

Whereas two research groups, Shen et al [11] and Yagi et al [12], reported three epigenetic subtypes and some genes as hypermethylation markers, Hinoue et al. identified four subtypes based on hierarchical clustering of DNA methylation at loci exhibiting high inter-tumor variability [13]. Two loci, representing CIMP-H and CIMP-L tumors, were associated with BRAF and KRAS mutations, respectively. Tumors in the third cluster were associated with TP53 mutations and prevalence in the distal colon, while the fourth cluster was enriched for tumors from the rectum, with low rates of KRAS and TP53 mutations. Moreover, previous studies have suggested that differences in the CIMP status are associated with differences in the transcriptomic level across several tumor types [8].

Using bioinformatics approach to select and validate markers aberrantly methylated in CRC has been attempted many times. Integration of epigenomics and genomics data identified 27 genes with hypermethylation/down-regulation, of which ADHFE1, BOLL, SLC6A15 and TFPI2, and TFPI2, EYA4, NPY, TWIST1, LAMA1 and GAS7 were experimentally validated [14, 15]. Another study suggested 8 genes, ADHFE1, C1orf70, SND1, OPLAH, TLX2, ZFP64, NR5A2 and COLAA with diagnostic potential in CRC [16].

Our aim was also to identify new aberrantly methylated gene promoters and observe their expression. Our approach however was different. We used the data from TCGA, in which the DNA methylation experiment was done using microarrays, containing over 450.000 sites within the genome. Unsupervised clustering of methylation data resulted in four clusters and each was compared to the methylation data of normal mucosa samples. The aberrantly methylated probes were intersected among all clusters to obtain the probes common to all clusters. The common methylation sites in all clusters were integrated with gene expression analysis, to identify novel candidate biomarkers, some of which we tested on our experimental set of samples. Finding common epigenetic alterations in all CRC types, regardless of tumor stage, could be a starting point for testing these methylation changes on cfDNA obtained from patient's blood and/or novel therapeutic targets.

\section{Methods \\ Bioinformatics methods \\ Patients and data}

Colon adenocarcinoma (COAD) and rectum adenocarcinoma (READ) data were obtained from The Cancer Genome Atlas (TCGA). Data were downloaded from the Broad GDAC Firehose portal (https://gdac.broadinstitute.org/) and contained clinical information, methylation, gene expression and mutation data. Platform used for DNA methylation experiment was Illumina Infinium HumanMethylation450k BeadChip array (HM450), which covers 482,421 CpG sites within the human genome. For methylation analysis level 3 data was used, which is already normalized and contains beta-value calculations, genomic coordinate, chromosome number and HUGO gene symbol for each CpG site on the array. For gene expression analysis mRNAseq experiment performed on Illumina HiSeq platform was used. Gene expression levels were obtained through RNAseqV2 pipeline, which uses a combination of MApSplice and "scaled estimate" (RSEM) to determine expression levels. RNAseqV2 data contains a normalized read count, which represents normalized RSEM count estimates from the upper quartile. Mutation data was obtained through variant calling from DNAseq experiment using MuTect2 pipeline. There were 381 tumor samples with methylation data on HM450 platform and complete mutational profile. From these 381 samples, 359 samples had also Illumina mRNAseqV2 gene expression data. 
There were 45 normal samples used for comparison in methylation data and 51 normal samples used for comparison in the gene expression dataset.

\section{Probes and genes}

The coordinates of protein-coding genes were downloaded from Ensembl, release 89 (http://www.ensembl. org/). The nomenclature of genes was unified according to The HUGO Gene Nomenclature Committee (HGNC) (http://www.genenames.org/). We mapped the HM450 probes to the GRCh38/hg38 genome using recently published study [17]. Location of mapped probes were overlapped them with promoter regions of regulatory build of genome and assigned to their nearest genes. The genes where transcription start site was within $5 \mathrm{~kb}$ of the mapped promoter region were used for further analysis.

\section{Unsupervised clustering}

We used the recursively petitioned mixture model (RPMM) for the identification of colorectal tumor subgroups based on the HM450 DNA methylation data. RPMM is a model-based unsupervised clustering approach developed for beta-distributed DNA methylation measurements that lie between 0 and 1 and is implemented as the RPMM Bioconductor package [18]. We removed probes mapped on $\mathrm{X}$ and $\mathrm{Y}$ chromosome and the probes containing "NA" values and performed RPMM clustering on 4165 probes, that showed the most variable DNA methylation levels (standard deviation > 0.25). A fanny algorithm (a fuzzy clustering algorithm) was used for initialization and level-weighted version of Bayesian information criterion (BIC) as a split criterion for an existing cluster as implemented in the R-based RPMM package.

\section{Differentially methylated probes and differentially expressed genes}

Differentially methylated probes and differentially expressed genes were obtained using TCGAbiolinks package in R [19]. Differentially methylated probes were obtained by comparing beta-values of probes between each methylation cluster and probes in normal samples. First, the mean methylation of each group for each probe was calculated, second, $p$-value was calculated using Wilcoxon test using Bonferroni adjustment method. The cutoff parameters were set to: absolute difference in methylation was larger than 0.2 and adjusted p-value less than 0.01 . For obtaining differential gene expression general log-linearized model was used, with cutoff parameters: absolute fold change was larger than 1.0, and false discovery rate (FDR) adjusted p-value less than 0.01. For each cluster, we selected methylation probes mapping to promoter regions and had absolute methylation difference more than 0.3 compared to normal. We selected hypermethylated promoter probes (methylation difference more than 0.3) and down-regulated genes with logarithmic fold change of at least - 1.0. Our selection also included hypomethylated promoter probes (methylation difference was less than -0.3) and up-regulated genes with logarithmic log fold change more than 1.0. This selection was overlapped among all resulting clusters to obtain the genes with aberrant methylation and differential expression common to all four clusters.

\section{Data visualization, text mining and survival analysis}

The HM450 DNA methylation $\beta$-values of 4165 most variable probes along with methylation cluster, location, gender, tumor stage, $M L H 1$ promoter methylation and mutations in BRAF, KRAS, APC and TP53 were represented graphically using heatmap visualization from ComplexHeatmap package in $\mathrm{R}$ programming software [20]. For construction of protein-protein interaction networks the STRING database (version 10.5) was used which produces a functional association network, using interaction sources, such as text mining, experiments, database, co-expression, neighborhood, gene fusion and co/occurrence. To identify gene ontology processes enriched within our 198 set of genes from the intersection of all resulting clusters the STRING database was used [21]. We used the GeneRIF (Gene Reference into Function) database as the source text for finding gene-disease associations previously published and stored on PubMed system. We performed several queries using different conditions and terms such as: "cancer", "colorectal", "colon", "methylation", "expression" and identification numbers for all 198 genes. For the Cox proportional hazard model package survival in $\mathrm{R}$ software was used [22]. The influence of the different clinical and genetic parameters was determined with logrank test, where $p$-value was less than 0.05 . Some hazard ratios could not be computed, since gene was up/down regulated or hypo/hypermethylated in all samples. Hazard ration can be computed when there are two groups.

\section{Experimental validation Clinical samples}

Samples used for experimental validation in our study are presented in Table 1. Our study was comprised of 115 samples, of which 90 were fresh frozen tissue samples and 25 tissue samples were stabilized in RNAlater solution (Ambion). All the samples $(n=115)$ were used in the methylation experiment, however, the latter 25 samples, that were stored in RNAlater, were of sufficient quality to be used also for gene expression experiment. 
Table 1 Clinical data for samples used in validation

\begin{tabular}{|c|c|c|}
\hline & $\begin{array}{l}\text { Methylation set } \\
(n=115) \\
\mathrm{n}(\%)\end{array}$ & $\begin{array}{l}\text { Expression set } \\
(n=25) \\
n(\%)\end{array}$ \\
\hline \multicolumn{3}{|l|}{ Gender } \\
\hline Female & $54(47)$ & $10(40)$ \\
\hline Male & $61(53)$ & $15(60)$ \\
\hline \multicolumn{3}{|l|}{ Location } \\
\hline Ascending colon & $27(23.5)$ & $5(20)$ \\
\hline Transverse colon & $14(12.2)$ & $3(12)$ \\
\hline Descending colon & $6(5.2)$ & $1(4)$ \\
\hline Sigmoid colon & $18(15.7)$ & $3(12)$ \\
\hline Rectum & $50(43.5)$ & $13(52)$ \\
\hline \multicolumn{3}{|l|}{$\mathrm{T}$} \\
\hline $\mathrm{T} 1$ & $12(10.4)$ & $1(4)$ \\
\hline $\mathrm{T} 2$ & $28(24.3)$ & $3(12)$ \\
\hline T3 & $61(53.0)$ & $20(80)$ \\
\hline $\mathrm{T} 4$ & $14(12.2)$ & $1(4)$ \\
\hline \multicolumn{3}{|l|}{ N } \\
\hline NO & $56(48.7)$ & $15(60)$ \\
\hline $\mathrm{N} 1$ & $53(46.1)$ & $5(20)$ \\
\hline $\mathrm{N} 2$ & $5(4.3)$ & $4(16)$ \\
\hline$N x$ & $1(0.9)$ & $1(4)$ \\
\hline \multicolumn{3}{|l|}{ M } \\
\hline MO & $84(73.0)$ & $18(72)$ \\
\hline M1 & $13(11.3)$ & $2(8)$ \\
\hline$M x$ & $18(15.7)$ & $5(20)$ \\
\hline
\end{tabular}

$T$ tumor size, $N$ lymph node infiltration, $N x$ lymph node infiltration not determined, $M$ distant metastasis, $M x$ distant metastasis not determined, $n$ number of samples

The latter 25 samples were collected during surgical colectomy of patients diagnosed with primary colorectal adenocarcinoma. The patients' whose samples were collected had no other cancer than CRC, and no previous radio- or chemotherapy. From each patient tumor and normal sample was collected, where normal samples of healthy colon mucosa were collected at least $20 \mathrm{~cm}$ away from tumor site. Both tumor and normal mucosa samples were placed in RNAlater solution, which stabilizes tissue and enables DNA and RNA extraction. Samples were submerged in RNAlater and incubated for $24 \mathrm{~h}$ at $4{ }^{\circ} \mathrm{C}$ to allow the solution to penetrate through the sample. After incubation period, the samples were stored at $-20^{\circ} \mathrm{C}$.

For all 115 samples data about gender, tumor location, size, nodal infiltration, distant metastasis, and survival data was obtained from Cancer Registry of Slovenia. Patients enrolled in the study signed an informed consent form agreeing to participate in the study. The National
Medical Ethics Committee of the Republic of Slovenia approved this research.

\section{RNA/DNA isolation}

DNA and RNA from tissues stored in RNALater solution were isolated with All prep DNA/RNA Mini Kit (Qiagen), according to the manufacturer's recommendations. DNA and RNA quantity and quality were determined spectrophotometrically by NanoDrop ND-1000 (Thermo Fisher Scientific). DNA $(n=90)$ was isolated from fresh frozen samples with QIAamp DNA Mini Kit (Qiagen), according to the manufacturer's recommendations. DNA quantity and quality were determined spectrophotometrically by NanoDrop ND-1000 (Thermo Fisher Scientific).

\section{Bisulfite conversion and MS-HRM experiment}

After DNA extraction, $1000 \mathrm{ng}$ of DNA was used in bisulfite conversion with innuCONVERT Bisulfite Basic Kit (Analytik Jena AG). Twenty ng of bisulfite converted DNA was used in methylation-sensitive high resolution melting experiment (MS-HRM). Primers for MS-HRM were designed in Methyl Primer Express Software v1.0 (Thermo Fisher Scientific) (Additional file 1: Table S1) to amplify both, methylated and unmethylated DNA. Amplicon length was designed to cover the specific $\mathrm{CpG}$ sites in the 5' UTR region of selected genes differentially methylated from the bioinformatics analysis. For some genes one amplicon covers more than one CpG site. As controls, completely methylated and completely unmethylated commercially available bisulfite converted DNA (EpiTect PCR Control DNA Set, Qiagen) were used in each MS-HRM run, to help with assessment of methylation status of the samples. The amplification was performed using the following protocol: $2.00 \mu \mathrm{L}$ bisulphite converted DNA, $1.00 \mu \mathrm{L}$ of each primer, $0.50 \mu \mathrm{L}$ dNTP, $1.00 \mu \mathrm{L}$ HotStart Taq Plus Buffer (10x), $0.05 \mu \mathrm{L}$ HotStart Taq Plus Polymerase $(5 \mathrm{U} / \mu \mathrm{L})$, and $0.3 \mu \mathrm{L}$ Syto9 with Nuclease-free water to obtain a total PCR reaction volume of $10 \mu \mathrm{L}$. Optimized cycling protocol for HRM analysis on the Rotor-Gene Q (Qiagen) was preformed including: initial denaturation at $95^{\circ} \mathrm{C}$ for $5 \mathrm{~min}$; 45 times at $94^{\circ} \mathrm{C}$ for $15 \mathrm{~s}$, annealing temperature (Additional file 1: Table S1) for 30 $\mathrm{s}$, extension at $72{ }^{\circ} \mathrm{C}$ for $30 \mathrm{~s}$ (using Fluorescence data acquisition on the "HRM" channel at this step). HRM analysis was performed immediately after PCR under the following conditions: $60-99^{\circ} \mathrm{C}$ with $0.1{ }^{\circ} \mathrm{C}$ ramp rate. This step requires fluorescence data acquisition on the "HRM" channel. All amplifications were performed in duplicate, using Rotor-Gene Q (Qiagen), following the manufacturer's recommendations.

\section{Reverse transcription and $q P C R$ experiment}

Gene expression levels were determined using SYBR Green-based quantitative polymerase chain reaction 
(qPCR), which was performed on Rotor-Gene Q (Qiagen) detection system. All the reagents were from Qiagen, except where otherwise indicated. For investigated genes and four endogenous controls (ACTB, GAPDH, $R N N 18 S$, and RPL13A) in qPCR experiment, primers were all predesigned and used according to manufacturer's instructions (Qiagen) (Additional file 2: Table S2).

Total RNA (300 ng) was reverse transcribed using QuantiTect Reverse Transcription Kit according to manufacturer's instructions (Qiagen). The resulting cDNA was diluted 100-fold, and $3 \mu \mathrm{l}$ was used for each qPCR reaction in $10 \mu \mathrm{l}$ PCR master mix $(5 \mu \mathrm{l} 2 \mathrm{x}$ QuantiTect SYBR Green PCR Master Mix, $1 \mu \mathrm{l}$ of forward and $1 \mu \mathrm{l}$ of reverse primer). All the $\mathrm{qPCR}$ reactions were performed in duplicates or triplicates. The signal was collected at the endpoint of every cycle. Following amplification, melting curves analysis of PCR products were acquired on the SYBR channel using a ramping rate of 1 ${ }^{\circ} \mathrm{C} / 60$ s for $60-95^{\circ} \mathrm{C}$.

For expression calculation, geometrical average of threshold cycle $\left(\mathrm{C}_{\mathrm{t}}\right)$ of four endogenous controls $(A C T B$, GAPDH, RNN18S, and RPL13A) was subtracted from ct of investigated gene to obtain the difference of threshold cycles $\Delta \mathrm{C}_{\mathrm{t}}$. The comparative threshold cycles $\left(\Delta \Delta \mathrm{C}_{\mathrm{t}}\right)$ were obtained by subtracting $\Delta \mathrm{C}_{\mathrm{t}}$ of tumor sample from $\Delta \mathrm{C}_{\mathrm{t}}$ of paired normal sample. The comparative threshold cycle is comparable with $\log \mathrm{FC}$, which is used for easier comparison with bioinformatics data.

\section{Results}

\section{Study design}

The study consisted of two major parts - bioinformatics analysis and experimental validation (Fig. 1). Bioinformatics analysis was performed on samples from projects COAD and READ obtained from TCGA. Methylation data was collected by experiment with HM450, which is the most comprehensive methylation data collection available on TCGA. Methylation data were obtained on the HM450 platform of 381 tumor tissue samples, together with 45 normal samples. HM450 covers 482,421 CPG sites within the genome, which were mapped to regulatory regions that are likely to be involved in gene regulation: the open chromatin region, predicted enhancer region, predicted promoter, predicted promoter flanking region and transcription factor binding site. Altogether we obtained 190,920 probes located in 81,467 regulatory regions. Specifically, 14,718 probes mapped into the open chromatin region, 9513 into the enhancer, 122,576 into the promoter, 42,150 into the promoter flanking region, and 13,670 probes mapped into the transcription factor binding site. Some regulatory regions can overlap, so some probes belong to more than one regulatory region.
Unsupervised clustering on methylation data resulted in four clusters. Probes in samples of each cluster were compared to probes of normal samples to obtain significant differentially methylated probes for each cluster. Differentially methylated probes with significant $p$-values of each cluster were intersected among all clusters. The location of the intersected probes was compared to a list of 190,920 probes in regulatory regions, which resulted in 3513 probes that were both in intersect among clusters and in regulatory regions. Gene expression data of the same samples were available from an Illumina mRNAseq V2 experiment. From the set of 381 tumor samples used in methylation analysis, gene expression data were available for 359 tumor samples. The samples in gene expression of each cluster was then compared to gene expression of 51 normal samples. The intersect among all four clusters gave 2422 differentially expressed genes. Integrating methylation in regulatory regions and gene expression data, considering hypermethylation/ down-regulation and hypomethylation/up regulation combinations, resulted in 590 aberrantly methylated probes belonging to 198 genes. The resulting 590 aberrantly methylated probes belong to 373 regulatory regions, in which 72 probes are located in the open chromatin region, 5 probes in the enhancer, 439 probes in the promoter, 97 probes in the promoter flanking region and 66 probes in the transcription factor binding site.

The second part of the study consisted of experimental validation of six selected genes. For this purpose, we tested DNA methylation status on 115 tumor tissue samples, of which 25 were paired tumor and normal tissue samples of sufficient quality for both DNA methylation and gene expression experiment.

\section{Clustering of methylation data}

Unsupervised clustering analysis was performed on the methylation data of 381 samples from COAD and READ. The clustering resulted in four separate clusters, denoted CIMP-H, CIMP-L, Cluster 3 and Cluster 4, according to the names used in the literature [10] (Fig. 2). As established previously, CIMP-H CRCs have a higher rate of hypermethylated promoter of the $M L H 1$ gene and higher mutation rate in gene BRAF. Similarly, we found MLH1 hypermethylation is present in $49.2 \%$ of samples in CIMP-H but only 13 samples out of 222 (5.8\%) from the other three clusters combined (Table 1). Almost all mutations in gene $B R A F$ were found in the CIMP-H cluster (35.6\%), a few were found in CIMP-L (4.8\%) and Cluster 3 (3.2\%), while none were found in Cluster 4. Cluster CIMP-L was characterized by a high frequency of $K R A S$ mutations, with rare mutations in $B R A F$, and low rate of TP53 mutations. Indeed, the rate of KRAS mutations in this cluster was the highest 
Bioinformatics approach

The Cancer Genome Atlas projects: COAD and READ

Methylation

Illumina Infinium HumanMethylation450k BeadChip array (HM450)

\begin{tabular}{|c|}
\hline $\begin{array}{c}\text { Samples: } 381 \text { tumor, } \\
45 \text { normal }\end{array}$ \\
\hline Clustering \\
\hline
\end{tabular}

Four clusters:

CIMP-H, CIMP-L, Cluster 3, Cluster 4
482,421 CpG sites

Promoter mapping

190,920 probes

3,513 aberrantly methylated probes

\begin{tabular}{|c|}
\hline Expression \\
\hline \multicolumn{1}{|c|}{ Illumina mRNAseq V2 } \\
\hline $\begin{array}{c}\text { Samples: } 359 \text { tumor, } \\
51 \text { normal }\end{array}$ \\
\hline $\begin{array}{c}\text { Differential Expression } \\
P<0.01, \mid\end{array}$ \\
\hline
\end{tabular}

2,422 differentially expressed genes

590 aberrantly methylated probes belong to 198 differentially expressed genes

\begin{tabular}{|c|c|c|c|}
\hline Literature mining & Gene ontology & Pathway analysis & Protein-protein interactions \\
\hline
\end{tabular}

Experimental validation

\begin{tabular}{|c|c|c|c|}
\hline \multicolumn{2}{|c|}{ Methylation } & \multicolumn{2}{|c|}{ Expression } \\
\hline 115 tumor samples & 25 normal samples & 25 tumor samples & 25 normal samples \\
\hline \multicolumn{4}{|c|}{ RNA/DNA isolation } \\
\hline Bisulfite conversion & MS-HRM experiment & Reverse transcription & qPCR experiment \\
\hline Gene & \multicolumn{2}{|c|}{ Methylation } & Expression \\
\hline CEP55 & \multicolumn{2}{|c|}{ 囚 } & $\hat{v}$ \\
\hline FOXD3 & \multicolumn{2}{|c|}{$\widehat{ن}$} & Z \\
\hline FOXF2 & \multicolumn{2}{|c|}{$\hat{v}$} & \& \\
\hline GNAO1 & \multicolumn{2}{|c|}{ ن } & 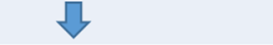 \\
\hline GRIA4 & \multicolumn{2}{|c|}{ ن } & $\sqrt{2}$ \\
\hline KCNA5 & \multicolumn{2}{|c|}{ ن } & そ \\
\hline
\end{tabular}

Fig. 1 Workflow of the study. Bioinformatics approach was composed of DNA methylation and gene expression analysis. DNA methylation data was obtained from experiment using Illumina Infinium HumanMethylation450k BeadChip (HM450) array from 381 tumor samples and 45 normal samples. Unsupervised clustering of tumor samples resulted in four clusters (CIMP-H, CIMP-L, Cluster 3 and Cluster 4). Probes of each cluster were compared to probes in normal samples group with Wilcoxon test to obtain differentially methylated probes. HM450 array contains 482,421 CpG sites, of which 190,920 CpG probes are located in promoter regions. The intersect of differentially methylated probes among all four methylation clusters and probes located in promoter region resulted in 3513 probes. Gene expression data was obtained from Illumina mRNAseq V2 experiment, which contains gene expression for 20,338 genes. From 381 tumor samples used in methylation analysis, 359 had gene expression data. For comparison of gene expression, 51 normal samples were used. Using gene expression data, tumor samples were divided into the same clusters as samples of methylation data and each cluster was compared to normal group using general log-linearized model to obtain differentially expressed genes. Intersect among all clusters in expression analysis gave 2422 differentially expressed genes. The bioinformatics approach resulted in 590 differentially methylated probes belonging to 198 differentially expressed genes, which exhibit hypermethylation/downregulation or hypomethylation/up regulation. After literature mining, gene ontology, pathway analysis and protein-protein interactions we selected CEP55, FOXD3, FOXF2, GNAO1, GRIA4 and KCNA5 for further experimental validation. For experimental validation we used 115 samples in methylation experiment and 25 samples in gene expression experiment. After RNA/DNA isolation, DNA was bisulfite converted and used in methylation-sensitive high-resolution melt experiment (MS-HRM), and RNA was reverse transcribed to CDNA and used in quantitative real-time PCR (qPCR) experiment. Gene CEP55 was hypomethylated and up regulated, while FOXD3, FOXF2, GNAO1, GRIA4 and KCNA5 were hypermethylated and down-regulated, consistent with our bioinformatics analysis. Figure was prepared in Microsoft Power Point software. Legend: COAD, Colon adenocarcinoma; READ, rectum adenocarcinoma; logFC, logarithm of fold change 


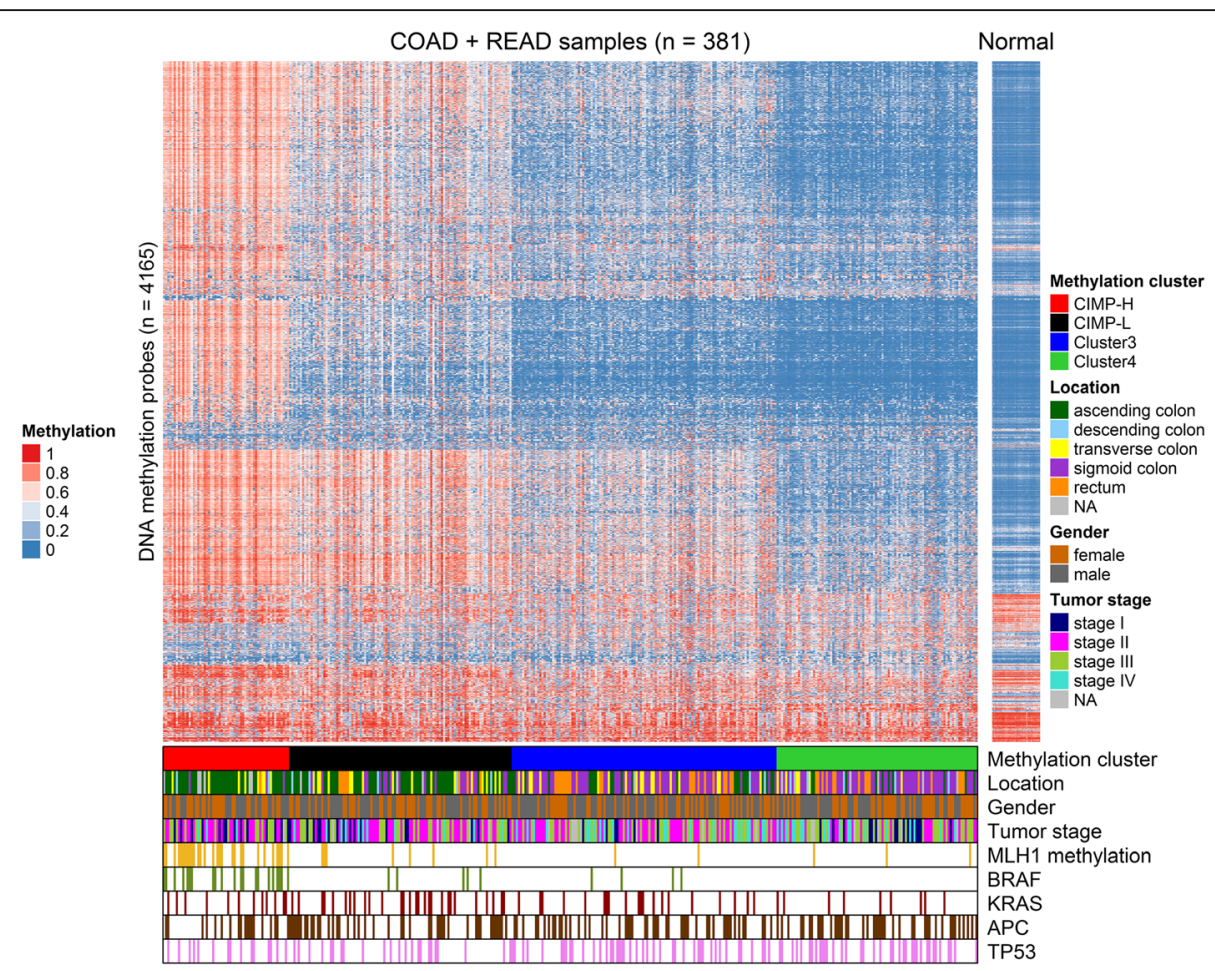

Fig. 2 Heatmap representing methylation, some clinical and genetic data. RPMM-based classification of 381 of tumor samples from colon adenocarcinoma (COAD) and rectum adenocarcinoma (READ) project in TCGA and heatmap representation of HM450 DNA methylation data. DNA methylation profiles of 4165 probes with most variable DNA methylation values (standard deviation > 0.25 ) in the 381 colorectal tumor sample set. The DNA methylation $\beta$-values are represented by a color scale from blue (low DNA methylation) to red (high DNA methylation). The probes are arranged based on the order of unsupervised hierarchal cluster analysis. Four subgroups were derived by RPMM-based clustering and are indicated below the heatmap: (red) CIMP-H cluster ( $n=59)$; (black) CIMP-L cluster $(n=104)$; (blue) Cluster 3 ( $n=124)$; (green) Cluster 4 ( $n=$ 94). Below methylation cluster is location: (dark green) ascending colon, (light blue) descending colon, (yellow) transverse colon, (purple) sigmoid colon, (orange) rectum, (grey) NA - data not available; gender: (light brown) female, (dark gray) male; tumor stage: (navy blue) stage I, (pink) stage II, (light green) stage III, (turquoise) stage IV, (gray) NA - data not available; MLH1 methylation (gold bars), BRAF mutation (olive bars), KRAS mutation (dark red bars), APC mutation (dark brown bars) and TP53 mutation (violet bars). On the right side of the heatmap are methylation levels of the samples from normal colorectal mucosa $(n=45)$ for comparison. The average age of patients is 64.2 and the average age of patients with normal mucosa is 68.8. Figure was prepared using library ComplexHeatmap in R programming software

(26.9\%) although not nearly as high as reported in the literature (92\%). Mutations in TP53 were found in 22.1\% of samples (Table 2). CIMP-H and CIMP-L clusters are both associated with tumor presence in ascending colon, where in our case, tumor presence in the ascending colon was $73.6 \%$ in CIMP-H and 52.5\% in CIMP-L.

The both non-CIMP clusters, Cluster 3 and Cluster 4, had lower frequencies in mutations in BRAF (3.2 and $0 \%)$ and KRAS (16.1 and 9.6\%), respectively. There was a higher rate of TP53 mutations, 34.7\% in Cluster 3 and $39.4 \%$ in Cluster 4 . Both of these clusters had a higher rate of tumor presence in sigmoid colon, $27.6 \%$ of samples in Cluster 3 and $39.6 \%$ in Cluster 4 , and rectum, $37.4 \%$ in Cluster 3 and $34.1 \%$ in Cluster 4. High microsatellite instability was more pronounced in CIMP-H (47.5\%), while low microsatellite instability was most frequent in CIMP-L (22.1\%). Microsatellite stability was predominant in Cluster 3 (82.3\%) and Cluster 4 (77.4\%) but it is also quite high in CIMP-L cluster (65.4\%). Fig. 2 shows there is no distinct feature (i.e., mutation, promoter methylation) common to all samples in any cluster or common to all clusters.

\section{Aberrantly methylated probes and differentially expressed genes}

Our analysis resulted in 590 aberrantly methylated probes found at the intersect between clusters and mapped to regulatory regions. These probes belong to 198 differentially expressed genes, which were differentially expressed in each cluster when compared to normal tissue samples (Additional file 3: Table S3). Using these 198 protein-coding genes, we performed protein-protein interaction network (PPIN), functional and literature mining analysis.

The 198 differentially expressed genes were uploaded to the STRING database to construct a PPIN (Additional file 4: Figure S1). Since genes were selected based on aberrant methylation and differential gene expression 
Table 2 Genetic and clinical features of all samples from TCGA and samples from TCGA belonging to each of the four methylationbased clusters

\begin{tabular}{|c|c|c|c|c|c|}
\hline & $\begin{array}{l}\text { CIMP-H }(n=59) \\
\text { n (\%) }\end{array}$ & $\begin{array}{l}\text { CIMP-L }(n=104) \\
\mathrm{n}(\%)\end{array}$ & $\begin{array}{l}\text { Cluster } 3(n=124) \\
\mathrm{n}(\%)\end{array}$ & $\begin{array}{l}\text { Cluster } 4(n=94) \\
\mathrm{n}(\%)\end{array}$ & $\begin{array}{l}\text { ALL }(n=381) \\
n(\%)\end{array}$ \\
\hline APC $/ W T$ & $19 / 40(32.2 / 67.8)$ & 43/61 (41.3/58.7) & 49/75 (39.5/60.5) & $42 / 52(44.7 / 55.3)$ & $153 / 228(40.2 / 59.8)$ \\
\hline KRAS/WT & $12 / 47(20.3 / 79.7)$ & 28/76 (26.9/73.1) & 20/104 (16.1/83.9) & 9/85 (9.6/90.4) & 69/312 (18.1/81.9) \\
\hline TP53/WT & $15 / 44(25.4 / 74.6)$ & 23/81 (22.1/77.9) & 43/81 (34.7/65.3) & $37 / 57(39.4 / 60.6)$ & 118/263 (31.0/69.0) \\
\hline BRAF/WT & 21/38 (35.6/64.4) & $5 / 99(4.8 / 95.2)$ & 4/120 (3.2/96.8) & 0/94 (0.0/100.0) & 30/351 (7.9/92.1) \\
\hline MLH1 Methylated/Unmethylated & 29/30 (49.2/50.8) & 8/96 (7.7/92.3) & $2 / 122(1.6 / 98.4)$ & $3 / 91(3.2 / 96.8)$ & 42/339 (11.0/89.0) \\
\hline Gender Female/Male & $30 / 29(50.8 / 49.2)$ & $46 / 58(44.2 / 55.8)$ & $51 / 73(41.1 / 58.9)$ & $47 / 47(50.0 / 50.0)$ & $174 / 207(45.7 / 54.3)$ \\
\hline \multicolumn{6}{|l|}{ Location } \\
\hline Ascending colon & $39(73.6)$ & $53(52.5)$ & $23(18.7)$ & $10(11.0)$ & $125(34.2)$ \\
\hline Transverse colon & $8(15.1)$ & $16(15.8)$ & $18(14.6)$ & $6(6.6)$ & $48(13.2)$ \\
\hline Descending colon & $2(3.8)$ & $5(5.0)$ & $2(1.6)$ & $5(5.5)$ & $14(3.8)$ \\
\hline Sigmoid colon & $3(5.7)$ & $13(12.9)$ & $34(27.6)$ & $36(39.6)$ & $86(23.6)$ \\
\hline Rectum & $1(1.9)$ & $14(13.9)$ & $46(37.4)$ & $31(34.1)$ & $92(25.2)$ \\
\hline No data & 6 & 3 & 1 & 3 & 16 \\
\hline \multicolumn{6}{|l|}{$\mathrm{T}$} \\
\hline $\mathrm{T} 1$ & $2(3.4)$ & $2(1.9)$ & $4(3.3)$ & $3(3.2)$ & $11(2.9)$ \\
\hline T2 & $12(20.3)$ & $18(17.3)$ & $7(5.7)$ & $15(16.1)$ & $52(13.7)$ \\
\hline T3 & $37(62.7)$ & $71(68.3)$ & $91(74.0)$ & $67(72.0)$ & $266(70.2)$ \\
\hline T4 & $7(11.9)$ & $13(12.5)$ & $21(17.1)$ & $8(8.6)$ & 49 (12.9) \\
\hline Tis & $1(1.7)$ & & & & $1(0.3)$ \\
\hline No data & & & 1 & 1 & 2 \\
\hline \multicolumn{6}{|l|}{ N } \\
\hline No & $38(64.4)$ & $63(60.6)$ & $60(48.8)$ & $43(46.2)$ & $204(53.8)$ \\
\hline N1 & $12(20.3)$ & $22(21.2)$ & $40(32.5)$ & $29(31.2)$ & $103(27.2)$ \\
\hline N2 & $9(15.3)$ & 19 (18.3) & $21(17.1)$ & $21(22.6)$ & $70(18.5)$ \\
\hline Nx & & & $2(1.6)$ & & $2(0.5)$ \\
\hline No data & & & 1 & 1 & 2 \\
\hline \multicolumn{6}{|l|}{ M } \\
\hline MO & $46(79.3)$ & $71(69.6)$ & $83(68.0)$ & $58(63.7)$ & $258(69.2)$ \\
\hline $\mathrm{M} 1$ & $3(5.2)$ & $11(10.8)$ & $23(18.9)$ & $16(17.6)$ & $53(14.2)$ \\
\hline$M x$ & $9(15.5)$ & 20 (19.2) & $16(13.1)$ & 17 (18.7) & 62 (16.6) \\
\hline No data & 1 & 2 & 2 & 3 & 8 \\
\hline \multicolumn{6}{|l|}{ MSI } \\
\hline MSI-H & $28(47.5)$ & $12(11.5)$ & $6(4.8)$ & $7(7.5)$ & $53(14.0)$ \\
\hline MSI-L & $9(15.3)$ & $23(22.1)$ & $16(12.9)$ & $14(15.1)$ & $62(16.4)$ \\
\hline MSS & $22(37.3)$ & $68(65.4)$ & $102(82.3)$ & $72(77.4)$ & $264(69.7)$ \\
\hline Indeterminate & & 1 & & 1 & 2 \\
\hline \multicolumn{6}{|l|}{ Stage } \\
\hline Stage I & $14(24.1)$ & $17(17.0)$ & $6(5.2)$ & $15(17.0)$ & $52(14.5)$ \\
\hline Stage II & $24(41.4)$ & $43(43.0)$ & $48(41.4)$ & $23(26.1)$ & 138 (38.1) \\
\hline Stage III & $17(29.3)$ & $29(29.0)$ & $40(34.5)$ & $32(36.4)$ & $118(32.6)$ \\
\hline Stage IV & $3(5.2)$ & $11(11.0)$ & $22(19.0)$ & $18(20.5)$ & $54(14.9)$ \\
\hline No data & 1 & 4 & 8 & 6 & 19 \\
\hline
\end{tabular}

CIMP the CpG island methylator phenotype, $n$ number of samples, MUT mutation, WT wild-type, MSI microsatellite instability, MSS microsatellite stable, $T$ tumor size, $N$ lymph node infiltration, $N x$ lymph node infiltration not determined, $M$ distant metastasis, $M x$ distant metastasis not determined 
present in all four clusters, some of the proteins coded by those genes are connected to networks, while others do not interact. To get some information about the biological functions of selected genes, we conducted gene ontology and pathway analysis (Additional file 5: Table S4). Pathway analysis reviled 12 significant KEGG pathways, of which the first three were neuroactive ligand-receptor interaction, cholinergic synapse and circadian entrainment. The circadian entrainment pathway has previously been associated with rectum adenocarcinoma [23]. We therefore selected two genes, GRIA4 and GNAO1, involved in this pathway, as well as gene KCNA5. KCNA5 is indirectly associated with this pathway through neighboring proteins KCNIP1 to NOS1. According to our PPIN analysis, there is another set of proteins with many interactions, of which BMP4 is the hub, which is involved in Hedgehog and TGF-beta signaling pathways. Moreover, our literature mining analysis revealed that the expression of gene BMP4 had already been studied (Additional file 6: Table S5), so we decided to select the genes FOXD3 and FOXF2 for experimental validation, whose proteins interact with BMP4 and both of which are transcription factors. We selected gene CEP55 on the basis of hypomethylation/up regulation, which is involved in biological process of cell cycle.

We constructed child PPIN with the six selected proteins described above, presented in Fig. 3, whereby b), c) and $\mathrm{d}$ ) were constructed using the neighboring proteins that are coded by genes from our list, and a) was constructed with the first interacted protein added, since CEP55 had no interactions in PPIN constructed from our gene set. Gene CEP55 is involved in cell division and the cell cycle process, GNAO1 and GRIA4 participate in signal transduction, FOXD3 and FOXF2 take part in stem cell differentiation and embryonic organ development, while KCNA5 is involved in negative regulation of cytosolic calcium ion concentration, protein oligomerization and action potential.

According to our in silico analysis, gene CEP55 was hypomethylated and up-regulated, while the other five genes, FOXD3, FOXF2, GNAO1, GRIA4 and KCNA5, were hypermethylated and down-regulated (Table 3). The genes expressed various levels of difference in methylation and expressions. The most down-regulated gene, regardless of cluster, was FOXD3 $(\operatorname{logFC}=-3.05)$. It was down-regulated in $98.3 \%$ of samples. The methylation difference was high (0.4), although present in fewer samples (91.9\%). The highest methylation difference was present in all four promoter probes of gene GRIA4 (from 0.46 to 0.54 ), with two of them present in 98.4\% of samples. Regardless of cluster, GRIA4 was down-regulated $(\log \mathrm{FC}=-2.41)$ in $98.1 \%$ of samples.

We evaluated the aberrant methylation of regulatory region per sample. In cases of GNAO1 and GRIA4, with which there was more than one methylation probe per gene, at least one of the probes had to be hypermethylated to conclude that the section was hypermethylated. No sample had less than two markers aberrantly methylated. There were two samples $(0.5 \%)$ with two markers and six samples (1.6\%) with three markers aberrantly methylated, $97.9 \%$ samples had at least four markers aberrantly methylated. Specifically, 33 samples (8.7\%) had four, 88 samples (23.1\%) had five and 252 samples (66.1\%) had all six markers aberrantly methylated.

To test the clinical data available in TCGA, we performed survival analysis using the Cox proportional hazards model (Table 4). Using univariate analysis, we found that late stage compared to early stage tumor has the highest hazard ratio. The second highest hazard ratio was observed with the presence metastasis, followed by presence of polyps and age above 60. By multivariate analysis, we obtained two significant hazard ratios, presence of metastasis and age above 60 . Overall model was significant, with $p$-value $2.475 \mathrm{e}-06$.

\section{Experimental validation}

Based on the bioinformatics analysis results described above, we experimentally validated six selected genes. Experimental validation of the methylation results of the in silico analysis was performed using a larger cohort of samples $(\mathrm{n}=115)$. Results revealed CEP55 to be hypomethylated in $97.3 \%$ of CRC cases and FOXD3, FOXF2, GNAO1, GRIA4 and KCNA5 being hypermethylated in $100,90.2,100,97.3$ and $99.1 \%$ of CRC cases, respectively.

The methylation and expression profile of 25 samples on which both experiments could be performed are shown on Fig. 4. Consistent with the bioinformatics analysis, our experimental data on expression analysis on 25 RNAlater stored samples showed an overall expression of gene CEP55 as up-regulated $(\operatorname{logFC}=7.47$, $p<0.001)$, while FOXD3 $(\log \mathrm{FC}=-0.66, p=0.027)$, FOXF2 $(\operatorname{logFC}=-1.33, p=0.021)$, GNAO1 $(\operatorname{logFC}=-$ 4.78, $p<0.001)$, GRIA4 $(\operatorname{logFC}=-3.25, p<0.001)$ and KCNA5 $(\log \mathrm{FC}=-2.81, p<0.001)$ were down-regulated in CRC compared to corresponding normal mucosa.

Methylation analysis on the same cohort of samples $(n=25)$ revealed that, in CRC compared to normal mucosa, gene CEP55 was completely hypomethylated and up-regulated. Gene GRIA4 had one sample hypomethylated and slightly up-regulated, all other samples were hypermethylated and down-regulated. Gene GNAO1 had one sample that was hypermethylated and up-regulated, all the other samples were hypermethylated and down-regulated. We obtained mixed results for FOXD3, FOXF2, and KCNA5 genes. FOXD3 had one sample hypomethylated and down-regulated, while seven samples were hypermethylated and up-regulated, the rest being hypermethylated and down-regulated. FOXF2 


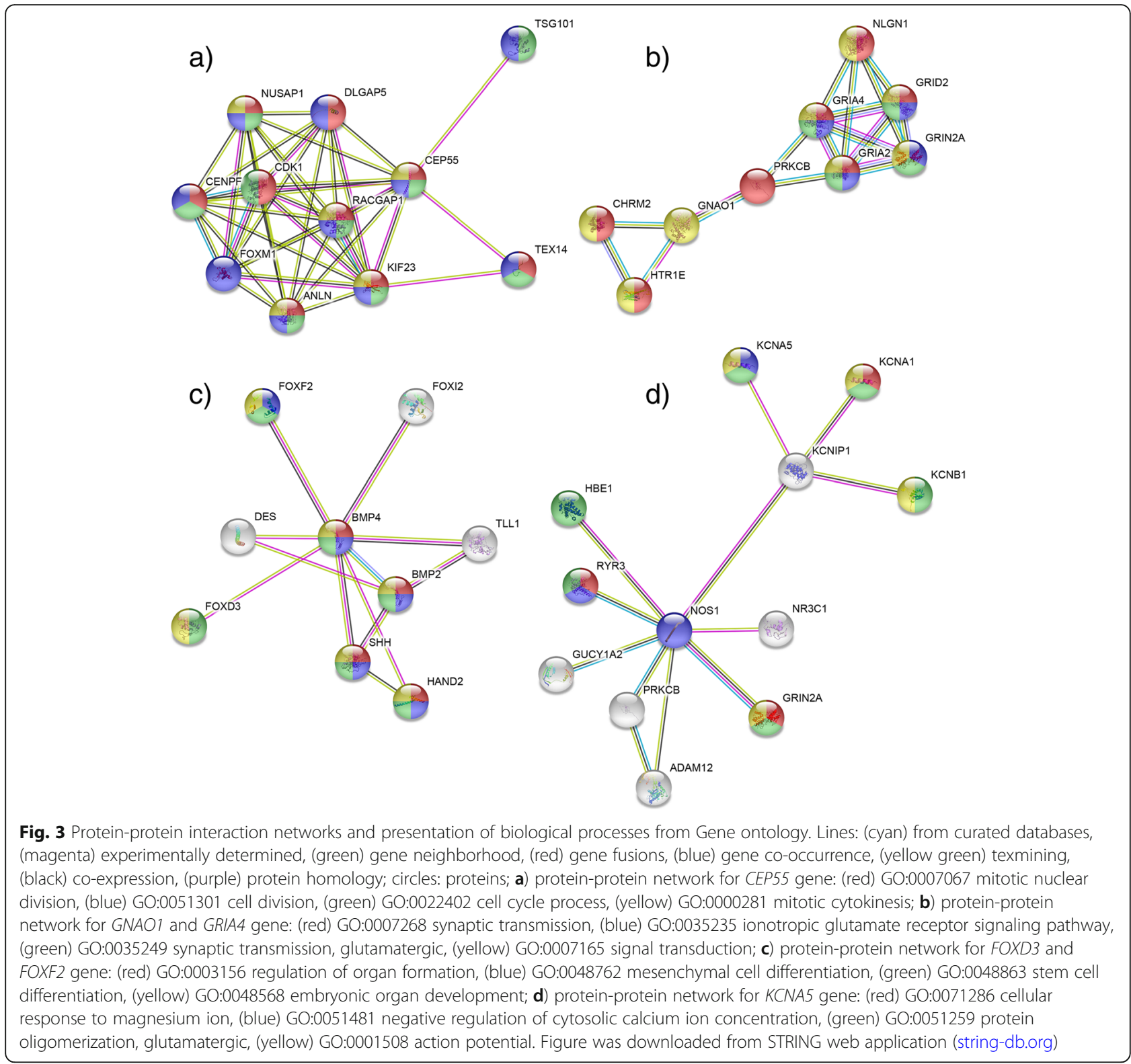

had two samples hypomethylated and up-regulated, four hypermethylated and up-regulated, while the others were hypermethylated and down-regulated. KCNA5 had no hypomethylated samples; four samples were hypermethylated and up-regulated, while the rest were hypermethylated and down-regulated.

Comparing the expression and methylation status of RNAlater stored samples $(n=25)$ showed that gene GNAO1 had the most down-regulated gene expression and was also hypermethylated in all samples (Table 5). Gene GRIA4 showed hypermethylation and down-regulation in 97.3 and $95.2 \%$ of samples, respectively. Up-regulation in all samples and hypomethylation in $97.3 \%$ of samples was observed in gene CEP55. The performance of other genes in terms of the correlation of their expression to methylation status was less encouraging, with FOXD3, FOXF2, and KCNA5 exhibiting down-regulation in fewer than $90 \%$ of samples, even only $68 \%$ for FOXF3.

Additionally, the number of aberrant methylation markers of six genes CEP55, FOXD3, FOXF2, GNAO1, GRIA4 and KCNA5 per sample was noted. There were no samples with fewer than three markers aberrantly methylated. Three samples $(2.6 \%)$ had three markers aberrantly methylated. There were $97.4 \%$ of samples with at least four markers aberrantly methylated. Specifically, 11 samples (9.6\%) had four, 22 samples (19.1\%) had five and 79 samples $(68.7 \%)$ had all six markers aberrantly methylated.

To test our clinical data, we performed survival analysis using the Cox proportional hazards model (Table 6). 


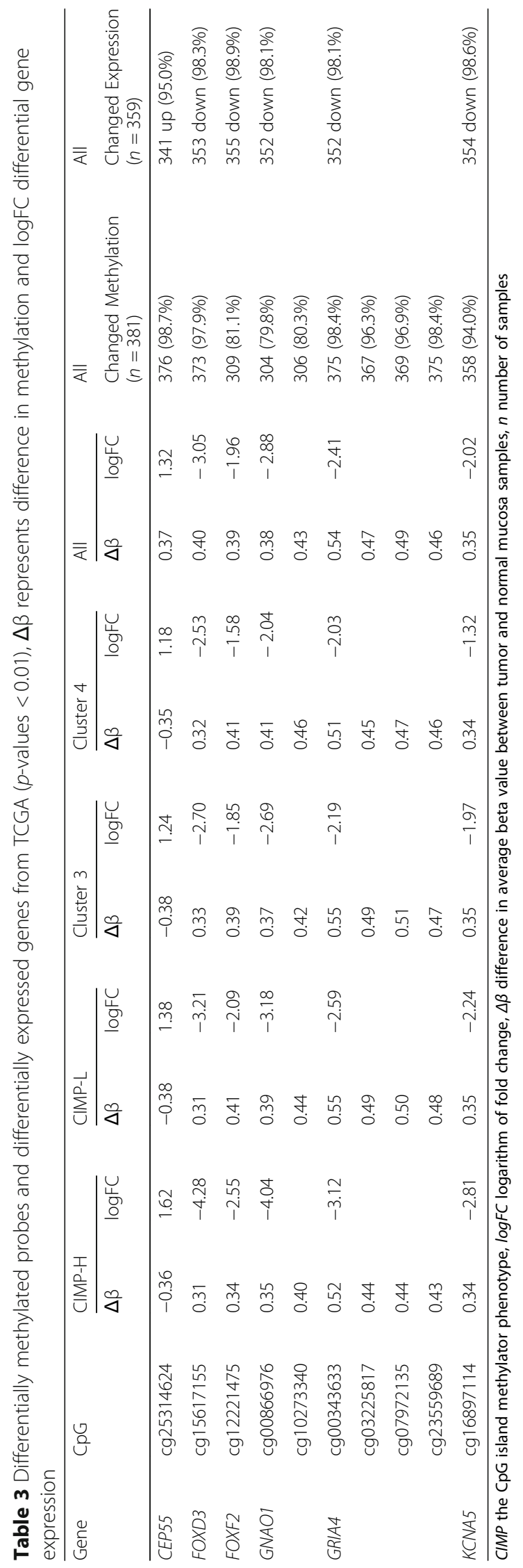


Table 4 Cox proportional hazards model on TCGA dataset

\begin{tabular}{|c|c|c|c|c|c|c|}
\hline & \multicolumn{3}{|c|}{ Univariate analysis } & \multicolumn{3}{|c|}{ Multivariate analysis } \\
\hline & hazard ratio & $\begin{array}{l}95 \% \text { confidence } \\
\text { interval }\end{array}$ & $p$-value & hazard ratio & $\begin{array}{l}95 \% \text { confidence } \\
\text { interval }\end{array}$ & $\begin{array}{l}p \text {-value ( } p \text {-value } \\
\text { of model) }\end{array}$ \\
\hline $\begin{array}{l}\text { Cluster (CIMP vs. } \\
\text { Non-CIMP }(n=180)\end{array}$ & 1.182 & $0.6763-2.065$ & 0.5593 & & & \\
\hline Age $(\geq 60)(n=180)$ & 2.328 & $1.193-4.544$ & 0.007842 & $3.693(n=155)$ & $1.634-8.349$ & $0.00169(2.475 e-06)$ \\
\hline Polyps present $(n=138)$ & 2.518 & $1.03-6.155$ & 0.03877 & & & \\
\hline $\begin{array}{l}\text { Gender (Male vs. } \\
\text { Female) }(n=180)\end{array}$ & 1.662 & $0.930-2.967$ & 0.07991 & & & \\
\hline $\begin{array}{l}\text { Location (rectum vs. } \\
\text { Colon) }(n=176)\end{array}$ & 1.07 & $0.4935-2.318$ & 0.8656 & & & \\
\hline $\begin{array}{l}\text { Stage (III/IV vs. I/II) } \\
(n=176)\end{array}$ & 1.95 & $1.073-3.543$ & 0.0251 & & & \\
\hline $\begin{array}{l}\text { Metastasis presence } \\
(n=155)\end{array}$ & 3.398 & $1.82-6.343$ & 0.0002934 & $3.813(n=155)$ & $2.032-7.156$ & $3.08 \mathrm{e}-05(2.475 \mathrm{e}-06)$ \\
\hline $\begin{array}{l}\text { Lymph node infiltration } \\
(n=179)\end{array}$ & 1.725 & $0.978-3.042$ & 0.05665 & & & \\
\hline $\begin{array}{l}\text { Tumor size }(\mathrm{T} 3 / \mathrm{T} 4 \mathrm{vs} \text {. } \\
\text { T1/T2) }(n=179)\end{array}$ & 1.746 & $0.6899-4.417$ & 0.206 & & & \\
\hline MSI vs. MSS ( $n=179)$ & 1.076 & $0.5948-1.947$ & 0.8092 & & & \\
\hline $\begin{array}{l}\text { Late stage vs. early stage } \\
(n=164)\end{array}$ & 3.876 & $0.9389-16$ & 0.04349 & & & \\
\hline \multicolumn{7}{|c|}{ Methylation (methylated vs unmethylated) $(n=165)$} \\
\hline CEP55 - cg25314624 & 1.3444 & $0.3213-5.626$ & 0.6842 & & & \\
\hline FOXD3 - cg15617155 & ND & & & & & \\
\hline FOXF2 - cg12221475 & 1.893 & $0.8455-4.236$ & 0.1146 & & & \\
\hline GNAO1- cg00866976 & 0.8484 & $0.4304-1.673$ & 0.6347 & & & \\
\hline cg10273340 & 0.8404 & $0.4161-1.697$ & 0.6275 & & & \\
\hline GRIA4 - cg00343633 & 0.4816 & $0.1483-1.563$ & 0.2138 & & & \\
\hline cg03225817 & 0.6222 & $0.1924-2.012$ & 0.4238 & & & \\
\hline cg07972135 & 0.6185 & $0.2206-1.734$ & 0.3564 & & & \\
\hline cg23559689 & 0.6887 & $0.1662-2.853$ & 0.605 & & & \\
\hline KCNA5 - cg16897114 & 1.91 & $0.4558-8.002$ & 0.3682 & & & \\
\hline \multicolumn{7}{|c|}{ Expression (upregulated vs down-regulated) } \\
\hline CEP55 & 1.089 & $0.3344-3.549$ & 0.8871 & & & \\
\hline FOXD3 & 1.859 & $0.4483-7.711$ & 0.3853 & & & \\
\hline FOXF2 & ND & & & & & \\
\hline GNAO1 & 0.9207 & $0.2222-3.815$ & 0.9092 & & & \\
\hline GRIA4 & 3.409 & 0.8084-14.38 & 0.07561 & & & \\
\hline KCNA5 & 1.859 & $0.4483-7.711$ & 0.3853 & & & \\
\hline
\end{tabular}

CIMP the CpG island methylator phenotype, $n$ number of samples, MSI microsatellite instability, MSS microsatellite stable, ND not determinable

Using univariate analysis, we found that the presence of metastasis had the highest hazard ratio, followed by cancer progression, both with the highest significance. The next two highest hazard ratios were tumor size and lymph node infiltration, with lower significance than the previous two. By multivariate analysis, we obtained four significant hazard ratios, presence of metastasis, age above 60, lymph node infiltration and cancer progression. The overall model had a $p$-value significance of $5.453 \mathrm{e}-04$.

\section{Discussion}

The vast database of experimental data (TCGA) was used in our bioinformatics study. Interestingly, we observed that some samples have no mutations in the most commonly mutated tumor suppressors and oncogenes 

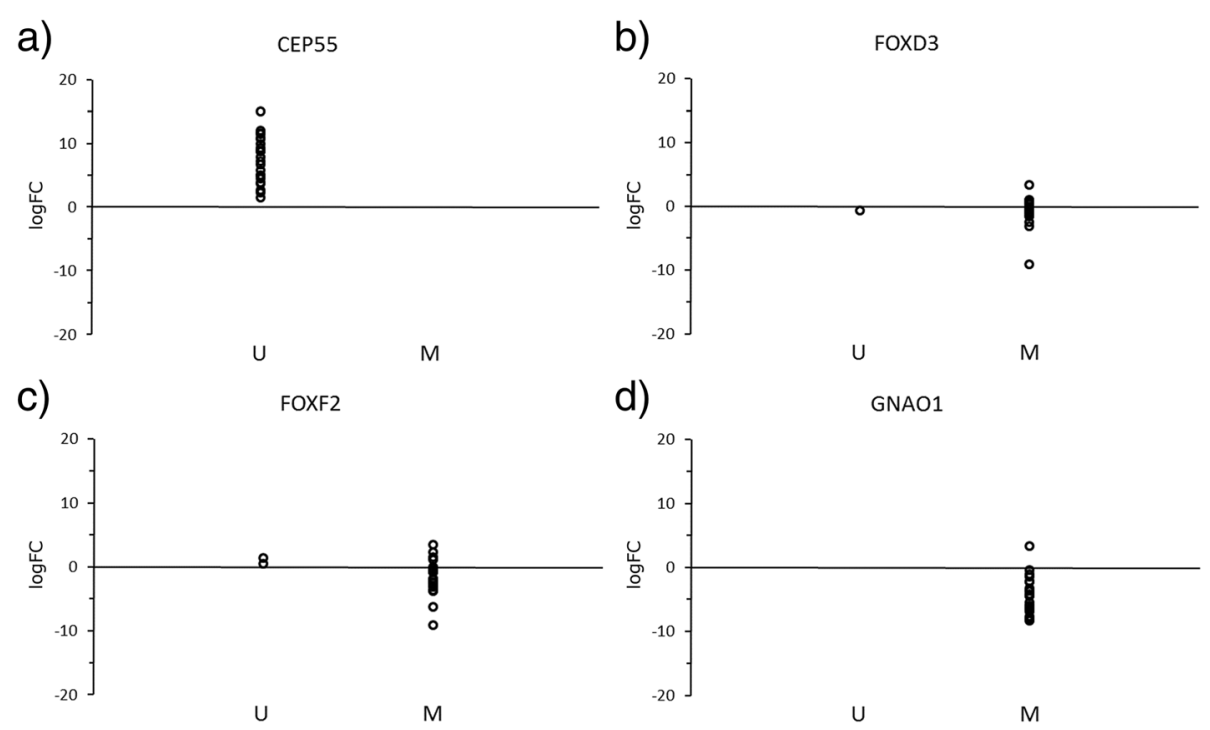

d) GNAO1
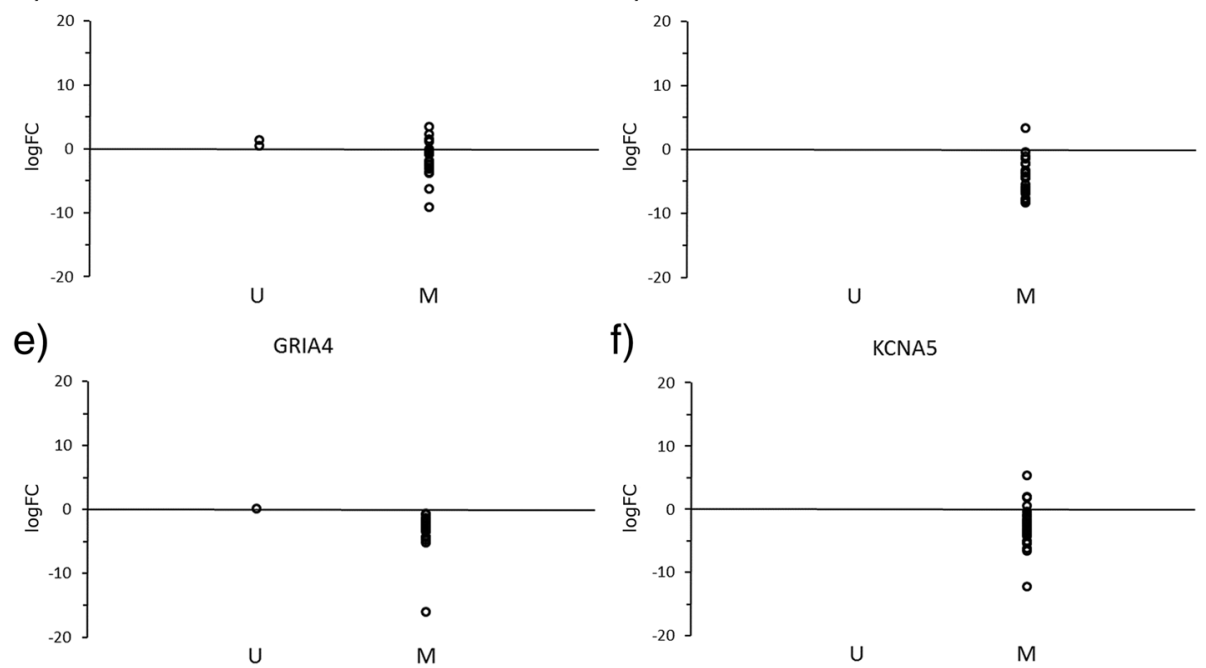

Fig. 4 Gene expression and methylation status of CEP55, FOXD3, FOXF2, GNAO1, GRIA4, and KCNA5 in our samples. For the 25 samples used in methylation and gene expression experiment, results are shown for gene: a) CEP55, b) FOXD3, c) FOXF2, d) GNAO1, e) GRIA4, and f) KCNA5. Figure was prepared in Microsoft Excel software. Legend: logFC, logarithm of fold change; U, unmethylated; M, methylated

$B R A F, K R A S$, TP53, APC, nor do they exhibit methylation in promoter of the $M L H 1$ gene. According to the data from the literature, only $30 \%$ of CRCs harbor the KRAS mutation, $8-15 \%$ of CRCs the BRAF mutation, $60 \%$ of CRCs the $A P C$ mutation, up to $40-50 \%$ of CRCs the TP53 mutation and $10-15 \%$ CRCs $M L H 1$ promoter methylation. Furthermore, we observed that neither $B R A F$ mutations nor methylation in the $M L H 1$ promoter can accurately describe the CIMP-H cluster, since a change in BRAF was present in roughly one third and
MLH1 methylation in roughly $50 \%$ of samples in $\mathrm{CIMP}-\mathrm{H}$. Both changes also had a small presence also in the other three clusters.

In search of a common epigenetic change, we performed bioinformatics study of methylation and expression analysis based on TCGA data, which resulted in 198 sets of genes. We performed pathway and gene ontology analysis on these genes. Pathway analysis resulted in 12 significant KEGG pathways, of which the first four were neuroactive ligand-receptor interaction, cholinergic synapse, circadian

Table 5 Methylation and gene expression on our dataset. $P$-value was calculated between $\Delta C_{t}$ of tumor and $\Delta C_{t}$ of normal mucosa samples

\begin{tabular}{|c|c|c|c|c|}
\hline \multirow[t]{2}{*}{ Gene } & \multicolumn{3}{|c|}{ Expression dataset $(n=25)$} & \multirow{2}{*}{$\begin{array}{l}\text { Methylation dataset }(n=115) \\
\text { Methylation }\end{array}$} \\
\hline & Methylation & Expression & $\operatorname{logFC}(p$-value) & \\
\hline CEP55 & 100\% Unmethylated & 100\% up-regulated & $7.47(<0.001)$ & 97.3\% unmethylated \\
\hline FOXD3 & $96 \%$ methylated & $68 \%$ down-regulated & $-0.66(0.027)$ & $100 \%$ methylated \\
\hline FOXF2 & $88 \%$ methylated & 76\% down-regulated & $-1.33(0.021)$ & $90.2 \%$ methylated \\
\hline GNAO1 & $100 \%$ methylated & 96\% down-regulated & $-4.78,(<0.001)$ & $100 \%$ methylated \\
\hline GRIA4 & $96 \%$ methylated & 95.2\% down-regulated & $-3.25(<0.001)$ & 97.3\% methylated \\
\hline KCNA5 & $100 \%$ methylated & 84\% down-regulated & $-2.81(<0.001)$ & 99.1\% methylated \\
\hline
\end{tabular}


Table 6 Cox proportional hazards model for our dataset. In each calculation number of samples is 115

\begin{tabular}{|c|c|c|c|c|c|c|}
\hline \multirow{2}{*}{$\begin{array}{l}n=115 \\
\text { Clinical data }\end{array}$} & \multicolumn{3}{|c|}{ Univariate analysis } & \multicolumn{3}{|c|}{ Multivariate analysis } \\
\hline & Hazard ratio & $\begin{array}{l}95 \% \text { confidence } \\
\text { interval }\end{array}$ & $p$-value & Hazard ratio & $\begin{array}{l}95 \% \text { confidence } \\
\text { interval }\end{array}$ & $\begin{array}{l}p \text {-value ( } p \text {-value } \\
\text { of model) }\end{array}$ \\
\hline Age $(\geq 60)$ & 1.967 & $1.01-3.833$ & 0.043 & 2.825 & $1.330-6.000$ & $0.007(8.737 e-12)$ \\
\hline Gender (Male vs. Female) & 1.544 & $0.982-2.426$ & 0.058 & & & \\
\hline Metastasis presence & 6.900 & $3.392-14.04$ & $8.982 \mathrm{e}-10$ & 6.692 & $3.137-14.278$ & 8.8e-07 (8.737e-12) \\
\hline Lymph node infiltration & 2.016 & $1.367-2.975$ & $3.366 \mathrm{e}-4$ & 2.234 & $1.352-3.691$ & $0.002(8.737 e-12)$ \\
\hline Tumor size (T3/T4 vs. T1/T2) & 1.867 & $1.143-3.048$ & 0.011 & & & \\
\hline Location (rectum vs. colon) & 1.797 & $1.139-2.836$ & 0.011 & 2.047 & $1.212-3.457$ & $0.007(8.737 e-12)$ \\
\hline Late stage vs. early stage & 1.926 & $1.172-3.165$ & 0.009 & & & \\
\hline RT & 1.134 & $0.534-2.409$ & 0.743 & & & \\
\hline Chemotherapy & 1.148 & $0.653-2.018$ & 0.632 & & & \\
\hline Cancer progression & 2.573 & $1.583-4.184$ & $8.385 e-05$ & & & \\
\hline \multicolumn{7}{|c|}{ Methylation (methylated vs unmethylated) } \\
\hline CEP55 & 1.276 & $0.312-5.217$ & 0.734 & & & \\
\hline FOXD3 & ND & & & & & \\
\hline FOXF2 & 1.335 & $0.579-3.077$ & 0.500 & & & \\
\hline GNAO1 & ND & & & & & \\
\hline GRIA4 & 0.339 & $0.106-1.092$ & 0.05729 & & & \\
\hline KCNA5 & 0.0704 & $0.009-0.572$ & 0.001 & & & \\
\hline \multicolumn{7}{|c|}{ Expression (upregulated vs down-regulated) } \\
\hline CEP55 & ND & & & & & \\
\hline FOXD3 & 1.664 & $0.485-5.702$ & 0.413 & & & \\
\hline FOXF2 & 0.267 & $0.034-2.086$ & 0.176 & & & \\
\hline GNAO1 & 4.277 & $0.4992-36.65$ & 0.148 & & & \\
\hline GRIA4 & 4.478 & $0.4999-40.11$ & 0.142 & & & \\
\hline KCNA5 & 3.699 & $0.9577-14.29$ & 0.042 & & & \\
\hline
\end{tabular}

ND not determinable

entrainment and calcium signaling pathway. These pathways included altogether 26 of our genes. Gene Ontology resulted in 137 significant biological processes, in which all the pathways together included 155 genes from our list. The first most significant biological process pathways were mostly related to the nervous system and its development in one part, and in the other part pathways were related to cell differentiation, adhesion and development. Gene ontology molecular function revealed that genes in our selected set are involved in receptor activity, whereas the gene ontology cellular component showed that most of genes in our set are part of plasma membrane.

Among prominent identified genes were CEP55, involved in the cell cycle process, FOXD3 and FOXF2, which are involved in stem cell differentiation, GNAO1 and GRIA4, which participate in signal transduction and $K C N A 5$, which is a part of the regulation of the calcium ion concentration. We experimentally validated these six genes on our own CRC tissue samples, confirming the prediction of expression and methylation status. Using both approaches, we found gene CEP55 to be hypomethylated and up-regulated, while the other five genes, FOXD3, FOXF2, GNAO1, GRIA4 and KCNA5, were hypermethylated and down-regulated. A number of studies have already described CEP55 as an overexpressed gene in cancer tissue samples. It maps to chromosomal regions 10q23 and encodes centrosome- and midbody-associated protein [24]. It is the latest member discovered in the centrosomal relative protein family and it has an important role in cell mitosis [25]. Overexpression of gene CEP55 has been observed in variety of solid tumors, including colon cancer [26], bladder cancer [27], hepatocellular carcinoma [28], gastric cancer [29], esophagus adenocarcinoma [30] and ovarian carcinoma [24]. A previous study reported overexpression of CEP55 in 60\% (9/15 samples) of CRC tissue samples [26]. Overexpression of CEP55 activates p21 and enhances the cell cycle transition. In contrast, the knockdown of CEP55 inhibits cell growth in gastric [29] and breast cancer [31]. Moreover, CEP55 has an important role in final stage division, which involves the separation 
of two daughter cells [25, 32]. Overexpression of CEP55 leads to an increase in the number of multinucleated cells and defect in cytokinesis, which may lead to tumorigenesis. In our set of genes, FOXD3 and FOXF2 have had a few studies performed on colon or gastric cancers. First, forkhead box D3 (FOXD3) was found to be a suppressor of colon cancer formation. While transcriptional repressor FOXD3 is expressed in many types of embryonic cells, its knockdown dramatically increases human colon cancer cell proliferation, affecting the EGFR-Ras-Raf-MEK-ERK signaling pathway [33]. Methylation in the promoter region of another tumor suppressor FOXF2 has previously been associated with shorter survival in gastric cancer patients. Through the FOXF2-IRF2BPL- $\beta$-catenin axis, FOXF2 inhibits Wnt signaling by binding to E3 ligate IRF2BPL promoter and up-regulates IRF2BPL, which interacts with $\beta$-catenin for its ubiquitination and degradation [34]. Methylation of both tumor suppressors, FOXD3 and $F O X F 2$, could be responsible for their down-regulation, thus disturbing their interaction with other proteins.

The second set of three genes, GNAO1, GRIA4 and $K C N A 5$, has been less researched, with only a few studies related to cancer. Gene GNAO1 was found to be overexpressed in $62.9 \%$ of patients with gastric cancer [35], while in our CRC tissue samples gene GNAO1 was down-regulated. An association had been shown between overexpressed gene GNAO1 and tumor size, tumor differentiation, TNM stage and poor prognosis. Their findings also demonstrated that knockdown of GNAO1 leads to reduced proliferation and promotes the apoptosis of gastric cancer cells [35]. However, statistical evaluation of an effect of methylation status or expression of gene GNAO1 on tumor size and TNM status in our case is impossible, since gene methylation was observed in the majority of samples. The second gene from this set, GRIA4, was the most methylated gene in our in silico study, with two probes being present in $98.4 \%$ of samples. Moreover, its down-regulation was experimentally confirmed in $98.1 \%$ of CRC tissue samples. A recently published study reported detecting a change in methylation in all CRC tissue samples, results similar to ours, and methylated cfDNA of GRIA4 in $68.5 \%$ of metastatic CRC patients [36]. Potassium voltage-gated channel subfamily A member 5 (KCNA5) is a protein coding gene involved in tumor cell proliferation in Ewing sarcoma [37], while its role in CRC is still unknown. In our study, gene KCNA5 was methylated in all studied samples, while its expression was decreased in $84 \%$ of our CRC tissue samples. Speculatively, as well as Ewing sarcoma, methylation of KCNA5 could be responsible for stable silencing of this gene in CRC, thus contributing to proliferation of tumor cells.

We were not able to perform survival analysis, since the majority of samples had either a hypermethylated or hypomethylated promoter region of validated gene. Since expression analysis was performed on a small cohort of samples $(n=25)$, it did not seem reasonable to do survival analysis, e.g., for gene FOXD3, with which $68 \%$ of tumors had down-regulation of these gene and the remaining $32 \%$ had either no change or up-regulation.

A limitation of the study was that there were 115 samples available for experimental methylation analysis and only 25 for experimental expression analysis. There were also small discrepancies when comparing the methylation statuses of the entire cohort of 115 samples and 25 samples (Table 4). The biggest difference in methylation status was observed in the FOXD3 gene, with which the discrepancy was $4 \%$. Methylation status of CEP55, FOXF2, GRIA4 and KCNA5, exhibited 2.7, 2.2, 1.3 and $0.9 \%$ discrepancy, while GNAO1 was methylated in all samples, so showing no discrepancy.

\section{Conclusions}

In summary, using bioinformatics on TCGA data followed by experimental validation we identified a set of six genes, CEP55, FOXD3, FOXF2, GNAO1, GRIA4 and $K C N A 5$, being differentially expressed in CRC compared to normal mucosa and whose expression seemed to be methylation dependent. The results of both approaches revealed that their change is frequent in CRC, regardless of their subtype, methylation clusters and the mutation status of CRC. As such, these six genes are believed to be an early event in human CRC carcinogenesis and to be potential CRC biomarkers.

\section{Additional files}

Additional file 1: Table S1. Primers used for quantitative PCR experiment. (DOCX $15 \mathrm{~kb}$ )

Additional file 2: Table S2. Aberrantly methylated and differentially expressed genes. A list of probes aberrantly methylated belonging to differentially expressed genes in each cluster when compared to normal tissue samples. (DOCX $15 \mathrm{~kb}$ )

Additional file 3: Table S3. Aberrantly methylated and differentially expressed genes. A list of probes aberrantly methylated belonging to differentially expressed genes in each cluster when compared to normal tissue samples. (TIF $15 \mathrm{~kb}$ )

Additional file 4: Figure S1. Protein-protein interaction network (PPIN) The PPIN was performed using 198 differentially expressed genes from our study. (XLSX $372 \mathrm{~kb}$ )

Additional file 5: Table S5. Primers used for methylation-sensitive high resolution melting experiment. (XLSX $36 \mathrm{~kb}$ )

Additional file 6: Table S6. Literature mining analysis for differentially expressed genes from our study. (XLSX $10 \mathrm{~kb}$ )

\section{Abbreviations}

COAD: Colon adenocarcinoma; READ: rectum adenocarcinoma; logFC: logarithm of fold change; MS-HRM: Methylation-sensitive high resolution melting; MSI: Microsatellite instable; MSI-H: Microsatellite instablehigh; MSI-L: Microsatellite instable-low; MSS: Microsatellite stable; qPCR: Quantitative polymerase chain reaction; TCGA: The Cancer Genome Atlas; CRC: colorectal cancer 


\section{Acknowledgments}

The authors acknowledge Alenka Matjašič, PhD and Andrej Zupan, PhD for their help on MS-HRM method. NH, DJS, and EB are sincerely grateful to Prof. Nina Zidar, M.D., and Prof. Jože Pižem, M.D., for creating a positive work environment.

\section{Funding}

$\mathrm{NH}, \mathrm{DJS}, \mathrm{EB}$, and DG acknowledge the financial support from the Slovenian Research Agency through research core funding No. P3-0054. NH acknowledges the financial support from the Slovenian Research Agency through project Z3-6797. The funders had no role in study design, data collection, analysis and interpretation of data, or in the writing of the manuscript.

\section{Availability of data and materials}

The datasets analyzed in this study are available from Broad Institute (https:// gdac.broadinstitute.org/). All data generated during this study are included in this published article and its supplementary information files.

\section{Authors' contributions}

$\mathrm{NH}$ : Concept, collection of data, bioinformatics analysis, experimental design, data analysis, writing; DJS: bioinformatics analysis, writing; ES: experimental design, data analysis; EB: experimental design, data analysis, writing; DG: patients collection, writing. All the authors have read and approved the manuscript.

\section{Ethics approval and consent to participate}

Patients enrolled in the study signed an informed consent form agreeing to participate in the study. The National Medical Ethics Committee of the Republic of Slovenia approved this research (approval reference number: 70/04/09).

\section{Consent for publication}

Not applicable.

\section{Competing interests}

The authors declare that they have no competing interests.

\section{Publisher's Note}

Springer Nature remains neutral with regard to jurisdictional claims in published maps and institutional affiliations.

\section{Author details}

'Institute of Pathology, Faculty of Medicine, University of Ljubljana, Korytkova 2, SI-1000 Ljubljana, Slovenia. ${ }^{2}$ Agricultural Institute of Slovenia, Hacquetova ulica 17, SI-1000 Ljubljana, Slovenia.

\section{Received: 5 November 2018 Accepted: 3 April 2019}

\section{Published online: 15 April 2019}

\section{References}

1. Ferlay J, Shin HR, Bray F, Forman D, Mathers C, Parkin DM. Estimates of worldwide burden of cancer in 2008: GLOBOCAN 2008. Int J Cancer. 2010; 127(12):2893-917.

2. Grady WM, Carethers JM. Genomic and epigenetic instability in colorectal cancer pathogenesis. Gastroenterology. 2008;135(4):1079-99.

3. Pino MS, Chung DC. The chromosomal instability pathway in colon cancer. Gastroenterology. 2010;138(6):2059-72.

4. Boland CR, Goel A. Microsatellite instability in colorectal cancer. Gastroenterology. 2010;138(6):2073-87 e3.

5. Weisenberger DJ, Siegmund KD, Campan M, Young J, Long TI, Faasse MA, et al. CpG island methylator phenotype underlies sporadic microsatellite instability and is tightly associated with BRAF mutation in colorectal cancer. Nat Genet. 2006;38(7):787-93.

6. Wong JJ, Hawkins NJ, Ward RL. Colorectal cancer: a model for epigenetic tumorigenesis. Gut. 2007;56(1):140-8.

7. Yamagishi $\mathrm{H}$, Kuroda $\mathrm{H}$, Imai Y, Hiraishi H. Molecular pathogenesis of sporadic colorectal cancers. Chin J Cancer. 2016;35:4.

8. Moarii M, Reyal F, Vert JP. Integrative DNA methylation and gene expression analysis to assess the universality of the $\mathrm{CpG}$ island methylator phenotype. Hum Genomics. 2015;9:26.
9. Ogino S, Kawasaki T, Kirkner GJ, Loda M, Fuchs CS. CpG island methylator phenotype-low (CIMP-low) in colorectal cancer: possible associations with male sex and KRAS mutations. J Mol Diagn. 2006;8(5):582-8.

10. Cancer Genome Atlas N. Comprehensive molecular characterization of human colon and rectal cancer. Nature. 2012;487(7407):330-7.

11. Shen L, Toyota M, Kondo Y, Lin E, Zhang L, Guo Y, et al. Integrated genetic and epigenetic analysis identifies three different subclasses of colon cancer. Proc Natl Acad Sci U S A. 2007;104(47):18654-9.

12. Yagi K, Akagi K, Hayashi H, Nagae G, Tsuji S, Isagawa T, et al. Three DNA methylation epigenotypes in human colorectal cancer. Clin Cancer Res. 2010;16(1):21-33.

13. Hinoue $T$, Weisenberger $D J$, Lange $C P$, Shen $H$, Byun HM, Van Den Berg $D$, et al. Genome-scale analysis of aberrant DNA methylation in colorectal cancer. Genome Res. 2012;22(2):271-82.

14. Kim YH, Lee HC, Kim SY, Yeom YI, Ryu KJ, Min BH, et al. Epigenomic analysis of aberrantly methylated genes in colorectal cancer identifies genes commonly affected by epigenetic alterations. Ann Surg Oncol. 2011;18(8): 2338-47.

15. Kok-Sin T, Mokhtar NM, Ali Hassan NZ, Sagap I, Mohamed Rose I, Harun R, et al. Identification of diagnostic markers in colorectal cancer via integrative epigenomics and genomics data. Oncol Rep. 2015;34(1):22-32.

16. Naumov VA, Generozov EV, Zaharjevskaya NB, Matushkina DS, Larin AK, Chernyshov SV, et al. Genome-scale analysis of DNA methylation in colorectal cancer using Infinium HumanMethylation450 BeadChips. Epigenetics. 2013;8(9):921-34.

17. Zhou W, Laird PW, Shen H. Comprehensive characterization, annotation and innovative use of Infinium DNA methylation BeadChip probes. Nucleic Acids Res. 2017;45(4):e22.

18. Houseman EA, Christensen BC, Yeh RF, Marsit CJ, Karagas MR, Wrensch M, et al. Model-based clustering of DNA methylation array data: a recursivepartitioning algorithm for high-dimensional data arising as a mixture of beta distributions. BMC Bioinformatics. 2008;9:365.

19. Colaprico A, Silva TC, Olsen C, Garofano L, Cava C, Garolini D, et al. TCGAbiolinks: an R/Bioconductor package for integrative analysis of TCGA data. Nucleic Acids Res. 2016;44(8):e71.

20. Gu Z, Eils R, Schlesner M. Complex heatmaps reveal patterns and correlations in multidimensional genomic data. Bioinformatics. 2016;32(18):2847-9.

21. Szklarczyk D, Franceschini A, Wyder S, Forslund K, Heller D, Huerta-Cepas J, et al. STRING v10: protein-protein interaction networks, integrated over the tree of life. Nucleic Acids Res. 2015;43(Database issue):D447-52.

22. Therneau TM, Grambsch PM. Modeling Survival Data: Extending the Cox Model. New York: Springer; 2000.

23. Hua Y, Ma X, Liu X, Yuan X, Qin H, Zhang X. Abnormal expression of mRNA, microRNA alteration and aberrant DNA methylation patterns in rectal adenocarcinoma. PLoS One. 2017;12(3):e0174461.

24. Zhang W, Niu C, He W, Hou T, Sun X, Xu L, et al. Upregulation of centrosomal protein 55 is associated with unfavorable prognosis and tumor invasion in epithelial ovarian carcinoma. Tumour Biol. 2016;37(5):6239-54.

25. Fabbro M, Zhou BB, Takahashi M, Sarcevic B, Lal P, Graham ME, et al. Cdk1/ Erk2- and PIk1-dependent phosphorylation of a centrosome protein, Cep55, is required for its recruitment to midbody and cytokinesis. Dev Cell. 2005; 9(4):477-88

26. Sakai M, Shimokawa T, Kobayashi T, Matsushima S, Yamada Y, Nakamura Y, et al. Elevated expression of C10orf3 (chromosome 10 open reading frame 3 ) is involved in the growth of human colon tumor. Oncogene. 2006;25(3):480-6.

27. Singh PK, Srivastava AK, Rath SK, Dalela D, Goel MM, Bhatt ML. Expression and clinical significance of Centrosomal protein 55 (CEP55) in human urinary bladder transitional cell carcinoma. Immunobiology. 2015;220(1): 103-8.

28. Chen CH, Lu PJ, Chen YC, Fu SL, Wu KJ, Tsou AP, et al. FL10540-elicited cell transformation is through the activation of PI3-kinase/AKT pathway. Oncogene. 2007;26(29):4272-83.

29. Tao J, Zhi X, Tian Y, Li Z, Zhu Y, Wang W, et al. CEP55 contributes to human gastric carcinoma by regulating cell proliferation. Tumour Biol. 2014;35(5):4389-99.

30. Jiang W, Wang Z, Jia Y. CEP55 overexpression predicts poor prognosis in patients with locally advanced esophageal squamous cell carcinoma. Oncol Lett. 2017;13(1):236-42.

31. Wang $Y$, Jin T, Dai $X$, Xu J. Lentivirus-mediated knockdown of CEP55 suppresses cell proliferation of breast cancer cells. Biosci Trends. 2016;10(1):67-73. 
32. Zhao WM, Seki A, Fang G. Cep55, a microtubule-bundling protein, associates with centralspindlin to control the midbody integrity and cell abscission during cytokinesis. Mol Biol Cell. 2006;17(9):3881-96.

33. Li K, Guo Q, Yang J, Chen H, Hu K, Zhao J, et al. FOXD3 is a tumo suppressor of colon cancer by inhibiting EGFR-Ras-Raf-MEK-ERK signal pathway. Oncotarget. 2017:8(3):5048-56.

34. Higashimori A, Dong Y, Zhang Y, Kang W, Nakatsu G, Ng SS, et al. Forkhead box F2 suppresses gastric cancer through a novel FOXF2-IRF2BPL-betacatenin signaling axis. Cancer Res. 2018.

35. Liu Z, Zhang J, Wu L, Liu J, Zhang M. Overexpression of GNAO1 correlates with poor prognosis in patients with gastric cancer and plays a role in gastric cancer cell proliferation and apoptosis. Int J Mol Med. 2014;33(3):589-96

36. Barault L, Amatu A, Siravegna G, Ponzetti A, Moran S, Cassingena A, et al. Discovery of methylated circulating DNA biomarkers for comprehensive non-invasive monitoring of treatment response in metastatic colorectal cancer. Gut. 2018:67(11):1995-2005.

37. Ryland KE, Hawkins AG, Weisenberger DJ, Punj V, Borinstein SC, Laird PW, et al. Promoter methylation analysis reveals that KCNA5 Ion Channel silencing supports Ewing sarcoma cell proliferation. Mol Cancer Res. 2016;14(1):26-34.

Ready to submit your research? Choose BMC and benefit from:

- fast, convenient online submission

- thorough peer review by experienced researchers in your field

- rapid publication on acceptance

- support for research data, including large and complex data types

- gold Open Access which fosters wider collaboration and increased citations

- maximum visibility for your research: over $100 \mathrm{M}$ website views per year

At $\mathrm{BMC}$, research is always in progress.

Learn more biomedcentral.com/submissions 